\title{
Global and blow-up solutions for quasilinear parabolic equations with a gradient term and nonlinear boundary flux
}

\section{Changjun Li, Lu Sun and Zhong Bo Fang*}

*Correspondence:

fangzb7777@hotmail.com

School of Mathematical Sciences,

Ocean University of China, Qingdao,

266100, P.R. China

\begin{abstract}
This work is concerned with positive classical solutions for a quasilinear parabolic equation with a gradient term and nonlinear boundary flux. We find sufficient conditions for the existence of global and blow-up solutions. Moreover, an upper bound for the 'blow-up time', an upper estimate of the 'blow-up rate' and an upper estimate of the global solution are given. Finally, some application examples are presented.

MSC: 35R45; 35K65; 34A12
\end{abstract}

Keywords: quasilinear parabolic equations; gradient term; boundary flux; blow-up; global solution

\section{Introduction}

In this paper, we consider the quasilinear parabolic equation with a gradient term

$$
(g(u))_{t}=\nabla \cdot(a(u) b(x) c(t) \nabla u)+f(x, u, q, t) \quad \text { in } D \times(0, T),
$$

subject to the nonlinear boundary flux and initial conditions

$$
\begin{aligned}
& \frac{\partial u}{\partial n}=h(x, t) r(u) \quad \text { on } \partial D \times(0, T) \\
& u(x, 0)=u_{0}(x) \quad \text { in } \bar{D} .
\end{aligned}
$$

Here $D \subset \mathbb{R}^{N}(N \geq 1)$ is a bounded domain with a smooth boundary $\partial D, \bar{D}$ is the closure of $D, q=|\nabla u|^{2}, n$ is the outer normal vector and $T$ is the maximum existence time of $u(x, t) . a(u) b(x) c(t), f(x, u, q, t)$ and $h(x, t) r(u)$ are nonlinear diffusion coefficient, reaction term and boundary flux, respectively. Let $\mathbb{R}^{+}=(0,+\infty), \overline{\mathbb{R}^{+}}=[0,+\infty)$, and suppose that the function $g(s) \in C^{2}\left(\mathbb{R}^{+}\right), g^{\prime}(s)>0$ for any $s>0, a(s) \in C^{2}\left(\mathbb{R}^{+}\right), b(x) \in C^{1}(\bar{D}), c(t) \in C^{1}\left(\mathbb{R}^{+}\right)$, $f(x, u, q, t) \in C^{1}\left(\bar{D} \times \mathbb{R}^{+} \times \overline{\mathbb{R}^{+}} \times \overline{\mathbb{R}^{+}}\right)$is a nonnegative function, $h(x, t) \in C^{1}(\bar{D} \times(0, T))$, $r(s) \in C^{2}\left(\mathbb{R}^{+}\right)$is a positive function, and the positive function $u_{0}(x) \in C^{2}(\bar{D})$ satisfies the compatibility conditions. Under these assumptions, the classical parabolic equation theory [1, Section 3] ensures that there exists a unique classical solution $u(x, t)$ to problem (1.1)-(1.3) for some $T>0$, and the solution is positive over $\bar{D} \times[0, T)$. Moreover, by the regularity theorem $[2$, Chapter 3$]$, we know $u \in C^{3}(D \times(0, T)) \cap C^{2}(\bar{D} \times(0, T))$.

O2014 Li et al.; licensee Springer. This is an Open Access article distributed under the terms of the Creative Commons Attribution License (http://creativecommons.org/licenses/by/2.0), which permits unrestricted use, distribution, and reproduction in any medium, provided the original work is properly cited. 
Equation (1.1) describes the diffusion of concentration of some Newtonian fluids through porous media or the density of some biological species in many physical phenomena and combustion theories (see $[3,4]$ ). The nonlinear Neumann boundary value condition (1.2) can be physically interpreted as the nonlinear radial law (see, e.g., [5, 6]).

In recent years the questions like blow-up and global solvability for nonlinear evolution equations have been investigated extensively by many authors. In particular, for the parabolic equations with a gradient term, we refer to [7-12] etc. For example, Souplet and Weissler [7] studied the semilinear parabolic equation

$$
u_{t}=\Delta u+f(u, \nabla u) \quad \text { in } D \times(0, T) \text {, }
$$

subject to the homogeneous Dirichlet boundary condition. By using the comparison principle and constructing a self-similar lower solution, they obtained sufficient conditions for global existence and blow-up solutions. Andreu [8] used a similar method to study the quasilinear parabolic equation

$$
u_{t}=\Delta u^{m}+f\left(u, \nabla u^{m}\right) \quad \text { in } D \times(0, T)
$$

Chen [9] considered the following semilinear parabolic equation:

$$
u_{t}=\Delta u+f(u)+g(u)|\nabla u|^{2} \quad \text { in } D \times(0, T)
$$

with the homogeneous Dirichlet boundary condition. By estimating the integral of ratio of one solution to the other, the author proved both global existence and blow-up results. Then he used the same method to study a more generalized equation with a gradient term, see $[10]$.

For the nonlinear parabolic equations with Neumann boundary conditions, Lair and Oxley [11] considered the quasilinear parabolic equation without a gradient term

$$
u_{t}=\nabla \cdot(a(u) \nabla u)+f(u) \quad \text { in } D \times(0, T)
$$

subject to the homogeneous Neumann boundary conditions, and they obtained the necessary and sufficient conditions for the global existence and blow-up solution by the approximation method. Recently, Ding and Gao [12] investigated an initial boundary value problem of the quasilinear parabolic equation with a gradient term

$$
(g(u))_{t}=\Delta u+f\left(x, u,|\nabla u|^{2}, t\right) \quad \text { in } D \times(0, T),
$$

subject to boundary flux $\frac{\partial u}{\partial n}=r(u)$, and they obtained sufficient conditions for the global existence and blow-up solution, the upper estimate of global solution and blow-up time.

Motivated by the above works, we construct an appropriate auxiliary function and use the Hopf maximum principle to study problem (1.1)-(1.3). The aim of this paper is to obtain sufficient conditions for the existence of blow-up and global solution, an upper bound for the 'blow-up time', an upper estimate of the 'blow-up rate' and an upper estimate of the global solution and then to give some examples. 


\section{Main results and proof}

We now state and prove the main results of this paper. Firstly, we give sufficient conditions of the existence of a blow-up solution of problem (1.1)-(1.3).

Theorem 1 Let $u \in C^{3}(D \times(0, T)) \cap C^{2}(\bar{D} \times(0, T))$ be a solution of problem (1.1)-(1.3). Assume that the following conditions hold:

(1) For any $(x, s, q, t) \in \bar{D} \times \mathbb{R} \times \mathbb{R}^{+} \times \mathbb{R}^{+}$,

$$
a(s)>0, \quad b(x)>0, \quad c(t)>0, \quad r(s)>0, \quad h(x, t) \geq 0 ;
$$

(2) For any $(x, s, q, t) \in \bar{D} \times \mathbb{R}^{+} \times \mathbb{R}^{+} \times \mathbb{R}^{+}$,

$$
\begin{aligned}
& a^{\prime}(s) \geq 0, \quad h_{t}(x, t) \geq 0, \quad f_{q} \geq 0, \quad\left(\frac{a(s)}{g^{\prime}(s)}\right)^{\prime} \geq 0, \quad r^{\prime}(s) \geq \frac{a^{\prime}(s)}{a(s)} r(s), \\
& r^{\prime \prime}(s) \geq \frac{a^{\prime}(s)}{a(s)} r^{\prime}(s), \\
& c^{\prime}(t) \geq 0, \quad g^{\prime}(s)>0, \quad f_{t}(x, s, q, t) \geq \frac{c^{\prime}(t)}{c(t)} f(x, s, q, t), \\
& f_{s}(x, s, q, t) \geq \frac{r^{\prime}(s)}{r(s)} f(x, s, q, t) ;
\end{aligned}
$$

(3) For any $x \in\left\{x \mid f\left(x, u_{0}, q_{0}, 0\right)=0, x \in \bar{D}\right\}$,

$$
\nabla\left(a\left(u_{0}\right) b(x) c(0) \nabla u_{0}\right) \geq 0
$$

(4) The constant

$$
\beta=\min _{D_{1}}\left\{\frac{a\left(u_{0}\right)}{g^{\prime}\left(u_{0}\right) r\left(u_{0}\right)}\left[\nabla\left(a\left(u_{0}\right) b(x) c(0) \nabla u_{0}\right)+f\left(x, u_{0}, q_{0}, 0\right)\right]\right\}>0,
$$

where $D_{1}=\left\{x \mid f\left(x, u_{0}, q_{0}, 0\right) \neq 0, x \in \bar{D}\right\} \neq \phi, q_{0}=\left|\nabla u_{0}\right|^{2}$

(5) The integration

$$
\int_{M_{0}}^{+\infty} \frac{a(s)}{r(s)} d s<+\infty, \quad \text { where } M_{0}=\max _{\bar{D}} u_{0}(x)
$$

then the solution $u(x, t)$ of system (1.1)-(1.3) must blow up in finite time $T$ and

$$
\begin{aligned}
& T \leq \frac{1}{\beta} \int_{M_{0}}^{+\infty} \frac{a(s)}{r(s)} d s, \\
& u(x, t) \leq \Phi^{-1}(\beta(T-t)),
\end{aligned}
$$

where $\Phi(z)=\int_{z}^{+\infty} \frac{a(s)}{r(s)} d s, z>0$, and $\Phi^{-1}$ is the inverse function of $\Phi$.

Proof Consider the auxiliary function

$$
\Psi=-\frac{1}{r(u)} u_{t}+\beta \frac{1}{a(u)} .
$$


We find that

$$
\begin{aligned}
\nabla \Psi= & \frac{r^{\prime}}{r^{2}} u_{t} \nabla u-\frac{1}{r} \nabla u_{t}-\beta \frac{a^{\prime}}{a^{2}} \nabla u, \\
\Delta \Psi= & \left(\frac{r^{\prime \prime}}{r^{2}}-2 \frac{\left(r^{\prime}\right)^{2}}{r^{3}}\right) q u_{t}+2 \frac{r^{\prime}}{r^{2}} \nabla u \cdot \nabla u_{t}+\frac{r^{\prime}}{r^{2}} u_{t} \Delta u-\frac{1}{r} \Delta u_{t} \\
& -\beta\left(\frac{a^{\prime \prime}}{a^{2}}-2 \frac{\left(a^{\prime}\right)^{2}}{a^{3}}\right) q-\beta \frac{\left(a^{\prime}\right)}{a^{2}} \Delta u,
\end{aligned}
$$

and

$$
\begin{aligned}
\Psi_{t}= & \frac{r^{\prime}}{r^{2}}\left(u_{t}\right)^{2}-\frac{1}{r}\left(u_{t}\right)_{t}-\beta \frac{\left(a^{\prime}\right)}{a^{2}} u_{t} \\
= & \frac{r^{\prime}}{r^{2}}\left(u_{t}\right)^{2}-\frac{1}{r}\left[\frac{1}{g^{\prime}}\left(a b c \Delta u+a^{\prime} b c q+a c \nabla b \cdot \nabla u+f\right)\right]_{t}-\beta \frac{\left(a^{\prime}\right)}{a^{2}} u_{t} \\
= & \frac{r^{\prime}}{r^{2}}\left(u_{t}\right)^{2}-\beta \frac{\left(a^{\prime}\right)}{a^{2}} u_{t}-\frac{1}{g^{\prime} r}\left(a^{\prime} b c u_{t} \Delta u+a b c^{\prime} \Delta u+a b c \Delta u_{t}+a^{\prime \prime} b c q u_{t}\right. \\
& +a^{\prime} b c^{\prime} q+2 a^{\prime} b c \nabla u \cdot \nabla u_{t} \\
& \left.+a^{\prime} c u_{t} \nabla b \cdot \nabla u+a c^{\prime} \nabla b \cdot \nabla u+a c \nabla b \cdot \nabla u_{t}+2 f_{q} \nabla u \cdot \nabla u_{t}+f_{t}+f_{u} u_{t}\right) \\
& +\frac{g^{\prime \prime}}{\left(g^{\prime}\right)^{2} r}\left(a b c \Delta u+a^{\prime} b c q+a c \nabla b \cdot \nabla u+f\right) u_{t} .
\end{aligned}
$$

Hence, from (2.11) and (2.12) we have

$$
\begin{aligned}
\frac{a b c}{g^{\prime}} \Delta & \Psi-\Psi_{t} \\
= & \left(\frac{a b c}{g^{\prime}} \frac{r^{\prime \prime}}{r^{2}}-2 \frac{a b c}{g^{\prime}} \frac{\left(r^{\prime}\right)^{2}}{r^{3}}+\frac{a^{\prime \prime} b c}{g^{\prime}} \frac{1}{r}-\frac{a^{\prime} b c}{r} \frac{g^{\prime \prime}}{\left(g^{\prime}\right)^{2}}\right) q u_{t} \\
& +\left(2 \frac{a b c}{g^{\prime}} \frac{r^{\prime}}{r^{2}}+2 \frac{a b c}{g^{\prime}} \frac{1}{r}+2 \frac{f_{q}}{g^{\prime}} \frac{1}{r}\right) \nabla u \cdot \nabla u_{t} \\
& +\left(\frac{a b c}{g^{\prime}} \frac{r^{\prime}}{r^{2}}+\frac{a b c}{g^{\prime}} \frac{1}{r}-\frac{a b c}{r} \frac{g^{\prime \prime}}{\left(g^{\prime}\right)^{2}}\right) u_{t} \Delta u+\left(\frac{a^{\prime} b c^{\prime}}{g^{\prime}} \frac{1}{r}-\beta \frac{a b c}{g^{\prime}} \frac{a^{\prime \prime}}{a^{2}}+2 \beta \frac{a b c}{g^{\prime}} \frac{\left(a^{\prime}\right)^{2}}{a^{3}}\right) q \\
& +\left(\frac{a^{\prime} b c^{\prime}}{g^{\prime}} \frac{1}{r}-\beta \frac{a b c}{g^{\prime}} \frac{a^{\prime}}{a^{2}}\right) \Delta u-\frac{r^{\prime}}{r^{2}}\left(u_{t}\right)^{2}+\beta \frac{a^{\prime}}{a^{2}} u_{t}+\frac{a^{\prime} c}{g^{\prime} r} u_{t} \nabla b \cdot \nabla u+\frac{a c^{\prime}}{g^{\prime} r} \nabla b \cdot \nabla u \\
& +\frac{f_{t}}{g^{\prime} r}+\frac{f_{u}}{g^{\prime} r} u_{t}-\frac{a c}{r} \frac{g^{\prime \prime}}{\left(g^{\prime}\right)^{2}} u_{t} \nabla b \cdot \nabla u-\frac{f}{r} \frac{g^{\prime \prime}}{\left(g^{\prime}\right)^{2}} u_{t} .
\end{aligned}
$$

Using (2.10) leads to

$$
\nabla u_{t}=-r \nabla \Psi-\beta \frac{a^{\prime} r}{a^{2}} \nabla u+\frac{r^{\prime}}{r} u_{t} \nabla u .
$$

Now substituting (2.14) into (2.13) yields

$$
\begin{aligned}
\frac{a b c}{g^{\prime}} & \Delta \Psi+\left(\frac{a c}{g^{\prime}} \nabla b+2 \frac{f_{q}}{g^{\prime}} \nabla u+2 \frac{b c}{g^{\prime}} \frac{(a r)^{\prime}}{r} \nabla u\right) \nabla \Psi-\Psi_{t} \\
& =\left(\frac{a b c}{g^{\prime}} \frac{r^{\prime \prime}}{r^{2}}+\frac{a^{\prime \prime} b c}{g^{\prime}} \frac{1}{r}+2 \frac{a^{\prime} b c}{g^{\prime}} \frac{r^{\prime}}{r^{2}}+2 \frac{f_{q}}{g^{\prime}} \frac{r^{\prime}}{r}-\frac{a^{\prime} b c}{r} \frac{g^{\prime \prime}}{\left(g^{\prime}\right)^{2}}\right) q u_{t}
\end{aligned}
$$




$$
\begin{aligned}
& +\left(\frac{a b c^{\prime}}{g^{\prime}} \frac{1}{r}-\beta \frac{a b c}{g^{\prime}} \frac{a^{\prime}}{a^{2}}\right) \Delta u \\
& +\left(\frac{a b c}{g^{\prime}} \frac{r^{\prime}}{r^{2}}+\frac{a b c}{g^{\prime}} \frac{1}{r}-\frac{a b c}{r} \frac{g^{\prime \prime}}{\left(g^{\prime}\right)^{2}}\right) u_{t} \Delta u+\left(\frac{a^{\prime} c}{g^{\prime} r}+\frac{a c}{g^{\prime}} \frac{r^{\prime}}{r^{2}}-\frac{a c}{r} \frac{g^{\prime \prime}}{\left(g^{\prime}\right)^{2}}\right) u_{t} \nabla b \cdot \nabla u \\
& +\left(\frac{a^{\prime} b c^{\prime}}{g^{\prime}} \frac{1}{r}-\beta \frac{a b c}{g^{\prime}} \frac{a^{\prime \prime}}{a^{2}}-2 \beta \frac{a b c}{g^{\prime}} \frac{a^{\prime}}{a^{2}} \frac{r^{\prime}}{r}-2 \beta \frac{f_{q}}{g^{\prime}} \frac{a^{\prime} r}{a}\right) q+\left(\beta \frac{a^{\prime}}{a^{2}}+\frac{f_{u}}{g^{\prime} r}-\frac{f}{r} \frac{g^{\prime \prime}}{\left(g^{\prime}\right)^{2}}\right) u_{t} \\
& -\frac{r^{\prime}}{r^{2}}\left(u_{t}\right)^{2}+\left(\frac{a c^{\prime}}{g^{\prime} r}-\beta \frac{a c}{g^{\prime}} \frac{a^{\prime}}{a^{2}}\right) \nabla b \cdot \nabla u+\frac{f_{t}}{g^{\prime} r} .
\end{aligned}
$$

In fact, from (1.1) we see that

$$
\Delta u=\frac{1}{a b c}\left(g^{\prime} u_{t}-a^{\prime} b c q-a c \nabla b \cdot \nabla u-f\right) .
$$

Thus combining (2.15) and (2.16), we arrive at

$$
\begin{aligned}
\frac{a b c}{g^{\prime}} \Delta & \Psi+\left(\frac{a c}{g^{\prime}} \nabla b+2 \frac{f_{q}}{g^{\prime}} \nabla u+2 \frac{b c}{g^{\prime}} \frac{(a r)^{\prime}}{r} \nabla u\right) \nabla \Psi-\Psi_{t} \\
= & \left(\frac{a b c}{g^{\prime}} \frac{r^{\prime \prime}}{r^{2}}+\frac{a^{\prime \prime} b c}{g^{\prime}} \frac{1}{r}+\frac{a^{\prime} b c}{g^{\prime}} \frac{r^{\prime}}{r^{2}}+2 \frac{f_{q}}{g^{\prime}} \frac{r^{\prime}}{r}-\frac{\left(a^{\prime}\right)^{2} b c}{a g^{\prime}} \frac{1}{r}\right) q u_{t} \\
& +\left(\beta \frac{\left(a^{\prime}\right)^{2} b c^{\prime}}{a^{2} g^{\prime}}-\beta \frac{a^{\prime \prime} b c}{a g^{\prime}}-2 \beta \frac{a^{\prime} b c}{a g^{\prime}} \frac{1}{r}-2 \beta \frac{a^{\prime} r}{a^{2}} \frac{f_{q}}{g^{\prime}}\right) q \\
& +\left(\frac{c^{\prime}}{c} \frac{1}{r}-\frac{f}{g^{\prime}} \frac{r^{\prime}}{r^{2}}-\frac{a^{\prime}}{a} \frac{f}{g^{\prime}} \frac{1}{r}+\frac{f_{u}}{g^{\prime} r}\right) u_{t}+\frac{f_{t}}{g^{\prime} r}-\frac{c^{\prime}}{c} \frac{f}{g^{\prime} r} \\
& +\beta \frac{a^{\prime}}{a^{2}} \frac{f}{g^{\prime}}+\left(\frac{a^{\prime}}{a} \frac{1}{r}-\frac{1}{r} \frac{g^{\prime \prime}}{\left(g^{\prime}\right)^{2}}\right)\left(u_{t}\right)^{2} .
\end{aligned}
$$

In view of (2.9), we have

$$
u_{t}=-r \Psi+\beta \frac{r}{a}
$$

If we substitute (2.18) into (2.17), then it is easy to obtain

$$
\begin{aligned}
\frac{a b c}{g^{\prime}} \Delta \Psi+\left(\frac{a c}{g^{\prime}} \nabla b+2 \frac{f_{q}}{g^{\prime}} \nabla u+2 \frac{b c}{g^{\prime}} \frac{(a r)^{\prime}}{r} \nabla u\right) \nabla \Psi \\
+\left\{\left[\left(\frac{a^{\prime} r}{a}\right)^{\prime}+r^{\prime \prime}\right] q \frac{a b c}{g^{\prime} r}+2 \frac{f_{q}}{g^{\prime}} \frac{r^{\prime}}{r} q+\frac{a r}{g^{\prime}}\left(\frac{f}{a r}\right)_{u}+\frac{c^{\prime}}{c}\right\} \Psi-\Psi_{t} \\
=\beta \frac{b c}{g^{\prime}}\left(\frac{r^{\prime \prime}}{r}-\frac{a^{\prime}}{a} \frac{r^{\prime}}{r}\right) q+2 \beta \frac{f_{q}}{g^{\prime}}\left(\frac{r^{\prime}}{a}-\frac{a^{\prime} r}{a^{2}}\right) q+\beta \frac{c^{\prime}}{a c}+\left(\frac{a^{\prime}}{a} \frac{1}{r}-\frac{1}{r} \frac{g^{\prime \prime}}{g^{\prime}}\right)\left(u_{t}\right)^{2} \\
\quad+\frac{1}{g^{\prime} r}\left(f_{t}-\frac{c^{\prime}}{c} f\right)+\beta \frac{1}{a g^{\prime}}\left(f_{u}-f \frac{r^{\prime}}{r}\right) \\
=\beta \frac{a b c}{g^{\prime} r}\left(\frac{r^{\prime}}{a}\right)^{\prime} q+2 \beta \frac{f_{q}}{g^{\prime}}\left(\frac{r}{a}\right)^{\prime} q+\beta \frac{c^{\prime}}{a c}+\frac{g^{\prime}}{a r}\left(\frac{a}{g^{\prime}}\right)^{\prime}\left(u_{t}\right)^{2} \\
\quad+\frac{c}{g^{\prime} r}\left(\frac{f}{c}\right)_{t}+\beta \frac{r}{a g^{\prime}}\left(\frac{f}{r}\right)_{u} .
\end{aligned}
$$


From assumptions (2.1)-(2.3), it follows that the right-hand side of (2.19) is nonnegative, i.e.,

$$
\begin{aligned}
& \frac{a b c}{g^{\prime}} \Delta \Psi+\left(\frac{a c}{g^{\prime}} \nabla b+2 \frac{f_{q}}{g^{\prime}} \nabla u+2 \frac{b c}{g^{\prime}} \frac{(a r)^{\prime}}{r} \nabla u\right) \nabla \Psi \\
& \quad+\left\{\left[\left(\frac{a^{\prime} r}{a}\right)^{\prime}+r^{\prime \prime}\right] q \frac{a b c}{g^{\prime} r}+2 \frac{f_{q}}{g^{\prime}} \frac{r^{\prime}}{r} q+\frac{a r}{g^{\prime}}\left(\frac{f}{a r}\right)_{u}+\frac{c^{\prime}}{c}\right\} \Psi-\Psi_{t} \geq 0 .
\end{aligned}
$$

Then from (2.4) and (2.5) we have

$$
\begin{aligned}
\max _{\bar{D}} \Psi(x, 0) & =\max _{\bar{D}}\left\{-\frac{1}{g^{\prime}\left(u_{0}\right) r\left(u_{0}\right)}\left[\nabla\left(a\left(u_{0}\right) b(x) c(0) \nabla u_{0}\right)+f\left(x, u_{0}, q_{0}, 0\right)\right]+\beta \frac{1}{a\left(u_{0}\right)}\right\} \\
& \leq 0 .
\end{aligned}
$$

And as we can see, an explicit calculation

$$
\begin{aligned}
\frac{\partial \Psi}{\partial n} & =\frac{r^{\prime}}{r^{2}} u_{t} \frac{\partial u}{\partial n}-\frac{1}{r} \frac{\partial u_{t}}{\partial n}-\beta \frac{a^{\prime}}{a^{2}} \frac{\partial u}{\partial n}=\frac{r^{\prime}}{r^{2}} h u_{t}-\frac{1}{r}(h r)_{t}-\beta \frac{a^{\prime}}{a^{2}} h r \\
& =\frac{r^{\prime}}{r^{2}} h u_{t}-h_{t}-\frac{r^{\prime}}{r} h u_{t}-\beta \frac{a^{\prime}}{a^{2}} h r=-h_{t}-\beta \frac{a^{\prime}}{a^{2}} h r \leq 0
\end{aligned}
$$

holds on $\partial D \times(0, T)$. Thus, by combining (2.20)-(2.22) and using the Hopf maximum principle, we find that the maximum of $\Psi$ on $\partial D \times(0, T)$ is 0 , i.e.,

$$
\Psi \leq 0 \quad \text { on } \partial D \times(0, T)
$$

and by (2.9), it gives

$$
\frac{a(u)}{r(u)} u_{t} \geq \beta
$$

Integrating (2.23) over $[0, t]$ at the point $x_{0} \in \bar{D}$, where $u_{0}\left(x_{0}\right)=M_{0}$, yields

$$
\frac{1}{\beta} \int_{M_{0}}^{u\left(x_{0}, t\right)} \frac{a(s)}{r(s)} d s \geq t
$$

This together with assumption (2.6) shows that $u(x, t)$ must blow up in finite time $T$; moreover,

$$
T \leq \frac{1}{\beta} \int_{M_{0}}^{+\infty} \frac{a(s)}{r(s)} d s
$$

For each fixed $x$, integrating inequality (2.23) over $[t, s](0<t<s<T)$ leads to

$$
\Phi(u(x, t)) \geq \Phi(u(x, t))-\Phi(u(x, s))=\int_{u(x, t)}^{u(x, s)} \frac{a(s)}{r(s)} d s \geq \beta(s-t)
$$

If we let $s \rightarrow T$, then formally

$$
\Phi(u(x, t)) \geq \beta(T-t)
$$


therefore

$$
u(x, t) \leq \Phi^{-1}(\beta(T-t)) .
$$

The proof is completed.

The result on the global solution is stated as Theorem 2 below.

Theorem 2 Let $u \in C^{3}(D \times(0, T)) \cap C^{2}(\bar{D} \times(0, T))$ be a solution of problem (1.1)-(1.3). Assume that the following conditions hold:

(1) For any $(x, s, q, t) \in \bar{D} \times \mathbb{R} \times \mathbb{R}^{+} \times \mathbb{R}^{+}$,

$$
a(s)>0, \quad b(x)>0, \quad c(t)>0, \quad r(s)>0, \quad h(x, t) \geq 0
$$

(2) For any $(x, s, q, t) \in \bar{D} \times \mathbb{R}^{+} \times \mathbb{R}^{+} \times \mathbb{R}^{+}$,

$$
\begin{aligned}
& a^{\prime}(s) \leq 0, \quad h_{t}(x, t) \leq 0, \quad f_{q} \leq 0, \quad\left(\frac{a(s)}{g^{\prime}(s)}\right)^{\prime} \leq 0, \\
& r^{\prime}(s) \geq \frac{a^{\prime}(s)}{a(s)} r(s), \quad r^{\prime \prime}(s) \leq \frac{a^{\prime}(s)}{a(s)} r^{\prime}(s), \\
& c^{\prime}(t) \leq 0, \quad g^{\prime}(s)>0, \quad f_{t}(x, s, q, t) \leq \frac{c^{\prime}(t)}{c(t)} f(x, s, q, t), \\
& f_{s}(x, s, q, t) \leq \frac{r^{\prime}(s)}{r(s)} f(x, s, q, t) ;
\end{aligned}
$$

(3) For any $x \in\left\{x \mid f\left(x, u_{0}, q_{0}, 0\right)=0, x \in \bar{D}\right\}$,

$$
\nabla\left(a\left(u_{0}\right) b(x) c(0) \nabla u_{0}\right) \geq 0
$$

(4) The constant

$$
\alpha=\max _{D_{1}}\left\{\frac{a\left(u_{0}\right)}{g^{\prime}\left(u_{0}\right) r\left(u_{0}\right)}\left[\nabla\left(a\left(u_{0}\right) b(x) c(0) \nabla u_{0}\right)+f\left(x, u_{0}, q_{0}, 0\right)\right]\right\}>0,
$$

where $D_{1}=\left\{x \mid f\left(x, u_{0}, q_{0}, 0\right)=0, x \in \bar{D}\right\} \neq \phi, q_{0}=\left|\nabla u_{0}\right|^{2}$;

(5) The integration

$$
\int_{m_{0}}^{+\infty} \frac{a(s)}{r(s)} d s<+\infty, \quad \text { where } m_{0}=\min _{\bar{D}} u_{0}(x)
$$

then the solution $u(x, t)$ of system (1.1)-(1.3) must be a global solution and

$$
u(x, t) \leq \Psi^{-1}\left(\alpha t+\Psi\left(u_{0}(x)\right)\right)
$$

where $\Psi(z)=\int_{m_{0}}^{z} \frac{a(s)}{r(s)} d s, z>0$, and $\Psi^{-1}$ is the inverse function of $\Psi$.

Proof Consider the auxiliary function

$$
\Phi=-\frac{1}{r(u)} u_{t}+\alpha \frac{1}{a(u)} .
$$


We first replace $\Psi$ and $\beta$ in (2.20) with $\Phi$ and $\alpha$, respectively, and under assumptions (2.26)-(2.28), we get

$$
\begin{aligned}
\frac{a b c}{g^{\prime}} \Delta \Phi-\left(\frac{a c}{g^{\prime}} \nabla b+2 \frac{f_{q}}{g^{\prime}} \nabla u+2 \frac{b c}{g^{\prime}} \frac{(a r)^{\prime}}{r} \nabla u\right) \nabla \Phi \\
\quad+\left\{\left[\left(\frac{a^{\prime} r}{a}\right)^{\prime}+r^{\prime \prime}\right] q \frac{a b c}{g^{\prime} r}+2 \frac{f_{q}}{g^{\prime}} \frac{r^{\prime}}{r} q+\frac{a r}{g^{\prime}}\left(\frac{f}{a r}\right)_{u}+\frac{c^{\prime}}{c}\right\} \Phi-\Phi_{t} \leq 0 .
\end{aligned}
$$

In fact, from (2.29) and (2.30) we can see that

$$
\begin{aligned}
\min _{\bar{D}} \Phi(x, 0) & =\min _{\bar{D}}\left\{-\frac{1}{g^{\prime}\left(u_{0}\right) r\left(u_{0}\right)}\left[\nabla\left(a\left(u_{0}\right) b(x) c(0) \nabla u_{0}\right)+f\left(x, u_{0}, q_{0}, 0\right)\right]+\alpha \frac{1}{a\left(u_{0}\right)}\right\} \\
& \geq 0 .
\end{aligned}
$$

Also, on $\partial D \times(0, T)$, it gives

$$
\frac{\partial \Phi}{\partial n}=-h_{t}-\alpha \frac{a^{\prime}}{a^{2}} h r \geq 0
$$

By combining (2.34)-(2.36) and using the Hopf maximum principle, we find that the minimum of $\Phi$ on $\partial D \times(0, T)$ is 0 , i.e.,

$$
\Phi \geq 0 \quad \text { in } \partial D \times(0, T)
$$

and by (2.33), we can see that

$$
\frac{a(u)}{r(u)} u_{t} \leq \alpha .
$$

For each fixed $x$, integrating $(2.37)$ over $[0, t]$ yields

$$
\frac{1}{\alpha} \int_{u_{0}(x)}^{u(x, t)} \frac{a(s)}{r(s)} d s \leq t
$$

This together with assumption (2.31) shows that $u(x, t)$ must be a global solution; moreover,

$$
\Psi(u(x, t))-\Psi\left(u_{0}(x)\right)=\int_{u_{0}(x)}^{u(x, t)} \frac{a(s)}{r(s)} d s \leq \alpha t,
$$

therefore

$$
u(x, t) \leq \Psi^{-1}\left(\alpha t+\Psi\left(u_{0}(x)\right)\right) .
$$

The proof is completed.

\section{Applications}

In what follows, we present several examples to demonstrate the applications of Theorems 1 and 2 . 
Example 1 Let $u$ be a solution of

$$
\begin{aligned}
& \left(e^{2 u}\right)_{t}=\nabla \cdot\left(e^{3 u}\left(1+\sum_{i=1}^{3} x_{i}^{2}\right) e^{t} \nabla u\right)+\left(1+\sum_{i=1}^{3} x_{i}^{2}\right) e^{4 u} q e^{t} \quad \text { in } D \times(0, T), \\
& \frac{\partial u}{\partial n}=2\left(1+t \sum_{i=1}^{3} x_{i}^{4}\right) e^{4 u} \quad \text { on } \partial D \times(0, T), \\
& u(x, 0)=u_{0}(x)=1+e^{4} \sum_{i=1}^{3} x_{i}^{2} \quad \text { in } \bar{D},
\end{aligned}
$$

where $D=\left\{x=\left(x_{1}, x_{2}, x_{3}\right) \mid \sum_{i=1}^{3} x_{i}^{2}<1\right\}$, then we have

$$
\begin{aligned}
& g(u)=e^{2 u}, \quad a(u)=e^{3 u}, \quad b(x)=1+\sum_{i=1}^{3} x_{i}^{2}, \quad c(t)=e^{t}, \\
& f(x, u, q, t)=\left(1+\sum_{i=1}^{3} x_{i}^{2}\right) e^{4 u} q e^{t}, \quad h(x, t)=2\left(1+t \sum_{i=1}^{3} x_{i}^{4}\right), \quad r(u)=e^{4 u} .
\end{aligned}
$$

It is easy to verify that (2.1)-(2.4) hold. By (2.5), we find

$$
\begin{aligned}
\beta & =\min _{D_{1}}\left\{\frac{a\left(u_{0}\right)}{g^{\prime}\left(u_{0}\right) r\left(u_{0}\right)}\left[\nabla\left(a\left(u_{0}\right) b(x) c(0) \nabla u_{0}\right)+f\left(x, u_{0}, q_{0}, 0\right)\right]\right\} \\
& =\min _{1 \leq u_{0}<1+e^{4}}\left\{\frac{1}{2}\left[3 u_{0}\left|\nabla u_{0}\right|^{2}+\left|\nabla u_{0}\right|^{2}+u_{0} \Delta u_{0}+e^{u_{0}} u_{0}\left|\nabla u_{0}\right|^{2}\right]\right\}=3 e^{4} .
\end{aligned}
$$

It follows from Theorem 1 that $u(x, t)$ must blow up in finite time $T$ and

$$
T \leq \frac{1}{\beta} \int_{M_{0}}^{+\infty} \frac{a(s)}{r(s)} d s=\frac{1}{\beta} \int_{2}^{+\infty} \frac{e^{3 s}}{e^{4 s}} d s=\frac{1}{3} e^{-6}
$$

and

$$
u(x, t) \leq \Phi^{-1}(\beta(T-t))=\ln \left[\frac{1}{3 e^{4}}(T-t)^{-1}\right]
$$

Example 2 Let $u$ be a solution of

$$
\begin{aligned}
& (u \sqrt{u})_{t}=\nabla \cdot\left(\frac{1}{\sqrt{u}}\left(1+\sum_{i=1}^{3} x_{i}^{2}\right) \frac{1}{1+t} \nabla u\right)+\left(1+\sum_{i=1}^{3} x_{i}^{2}\right) \frac{1-q}{1+t} \sqrt{u} \quad \text { in } D \times(0, T), \\
& \frac{\partial u}{\partial n}=\sqrt{2}\left(1+t \sum_{i=1}^{3} x_{i}^{4}\right)^{-1} \sqrt{u} \text { in } \partial D \times(0, T), \\
& u(x, 0)=u_{0}(x)=1+\sum_{i=1}^{3} x_{i}^{2} \quad \text { in } \bar{D}
\end{aligned}
$$


where $D=\left\{x=\left(x_{1}, x_{2}, x_{3}\right) \mid \sum_{i=1}^{3} x_{i}^{2}<1\right\}$, then we have

$$
\begin{aligned}
& g(u)=u \sqrt{u}, \quad a(u)=\frac{1}{\sqrt{u}}, \quad b(x)=\left(1+\sum_{i=1}^{3} x_{i}^{2}\right), \quad c(t)=\frac{1}{1+t}, \\
& f(x, u, q, t)=\left(1+\sum_{i=1}^{3} x_{i}^{2}\right) \frac{1-q}{1+t} \sqrt{u}, \quad h(x, t)=\sqrt{2}\left(1+t \sum_{i=1}^{3} x_{i}^{4}\right)^{-1}, \quad r(u)=\sqrt{u} .
\end{aligned}
$$

It is easy to verify that (2.26)-(2.29) hold. By (2.30), we find

$$
\begin{aligned}
\alpha & =\max _{D_{1}}\left\{\frac{a\left(u_{0}\right)}{g^{\prime}\left(u_{0}\right) r\left(u_{0}\right)}\left[\nabla\left(a\left(u_{0}\right) b(x) c(0) \nabla u_{0}\right)+f\left(x, u_{0}, q_{0}, 0\right)\right]\right\} \\
& =\max _{1 \leq u_{0}<2}\left\{\frac{1}{3}\left[-u_{0}^{-2}\left|\nabla u_{0}\right|^{2}+2 u_{0}^{-1} \Delta u_{0}+2\left(1-\left|\nabla u_{0}\right|^{-2}\right)\right]\right\}=\frac{14}{3} .
\end{aligned}
$$

It follows from Theorem 2 that $u(x, t)$ must be a global solution and

$$
u(x, t) \leq \Psi^{-1}\left(\alpha t+\Psi\left(u_{0}(x)\right)\right)=\exp \left(\alpha t+\ln u_{0}\right)=u_{0} \exp \left(\frac{14}{3} t\right)
$$

\section{Competing interests}

The authors declare that they have no competing interests.

\section{Authors' contributions}

All authors contributed equally to the manuscript and read and approved the final manuscript.

\section{Acknowledgements}

This work is supported by the Natural Science Foundation of Shandong Province of China (ZR2012AM018) and the Fundamental Research Funds for the Central Universities (No. 201362032). The authors would like to deeply thank all the reviewers for their insightful and constructive comments.

\section{Received: 27 February 2014 Accepted: 29 May 2014 Published: 05 Jun 2014}

\section{References}

1. Amann, H: Quasilinear parabolic systems under nonlinear boundary conditions. Arch. Ration. Mech. Anal. 92, 153-192 (1986)

2. Sperb, RP: Maximum Principles and Their Applications. Academic Press, New York (1981)

3. Bebernes, J, Eberly, D: Mathematical Problems from Combustion Theory. Springer, New York (1989)

4. Diaz, J, Thelin, FD: On a nonlinear parabolic problem arising in some model related to turbulent flows. SIAM J. Math. Anal. 25, 1085-1111 (1994)

5. Filo, J: Diffusivity versus absorption through the boundary. J. Differ. Equ. 99, 281-305 (1992)

6. Levine, HA, Payne, LE: Nonexistence theorems for the heat equation with nonlinear boundary conditions and for porous medium equation backward in time. J. Differ. Equ. 16, 319-334 (1974)

7. Souplet, P, Weissler, FB: Self-similar subsolutions and blowup for nonlinear parabolic equations. J. Math. Anal. Appl. 212, 60-74 (1997)

8. Andreu, F, Mazón, JM, Simondon, F, Toledo, J: Blow up for a class of nonlinear parabolic problems. Asymptot. Anal. 29(2), 143-155 (2002)

9. Chen, SH: Global existence and blowup of solutions for a parabolic equation with a gradient term. Proc. Am. Math. Soc. 129, 975-981 (2001)

10. Chen, $\mathrm{SH}, \mathrm{Yu}, \mathrm{DM}$ : Global existence and blowup solutions for quasilinear parabolic equations. J. Math. Anal. Appl. 335 , 151-167 (2007)

11. Lair, AV, Oxley, ME: A necessary and sufficient condition for global existence for degenerate parabolic boundary value problem. J. Math. Anal. Appl. 221, 338-348 (1998)

12. Ding, JT, Guo, BZ: Global existence and blow-up solutions for quasilinear reaction-diffusion equations with a gradient term. Appl. Math. Lett. 24, 936-942 (2011) 